\title{
Myeloperoxidase-Dependent Generation of Hypochlorite-Modified Proteins in Human Placental Tissues during Normal Pregnancy
}

\author{
Astrid Hammer, Gernot Desoye, Gottfried Dohr, Wolfgang Sattler, and Ernst Malle \\ Institute of Histology and Embryology (AH, GDo), Clinic of Obstetrics and Gynecology (GDe), and Institute of \\ Medical Biochemistry (WS, EM), Karl-Franzens University Graz, Graz, Austria
}

\begin{abstract}
SUMMARY: Myeloperoxidase (MPO), which is released from cytoplasmic granules of activated phagocytes by a degranulation process, reacts with $\mathrm{H}_{2} \mathrm{O}_{2}$ (generated during the oxidative burst) and chloride ions to generate hypochlorous acid/hypochlorite $\left(\mathrm{HOCl} / \mathrm{OCl}^{-}\right) . \mathrm{HOCl}$, a strong oxidant, in turn reacts with proteins to form $\mathrm{HOCl}$-modified proteins. The presence of these cytotoxic chloramines during inflammatory conditions, eg, atherosclerosis and glomerular and tubulointerstitial injury, suggested that chloramines are powerful oxidants that can have profound biologic effects. In the present study, immunoreactive MPO was identified in fetal membranes and the basal plate and in maternal and fetal blood cells of human placental tissues. Monocytes/macrophages represent the major cell source for MPO in human placental tissues. Immunohistochemical findings revealed that $\mathrm{HOCl}$-modified proteins are present in normal human term placenta but not during the first trimester of pregnancy (Weeks 7 to 12). HOCl-modified proteins were localized in areas formed by fetally derived cells as well as maternal decidual tissues, ie, areas where fetal extravillous trophoblast cells invade the maternal tissue and stimulate the maternal immune system. $\mathrm{HOCl}$-modified proteins, products of the $\mathrm{MPO}-\mathrm{H}_{2} \mathrm{O}_{2}$-chloride system in vivo, were not present intracellularly, but immunoreactivity for $\mathrm{HOCl}$-modified proteins was cell-associated and/or present in the extracellular matrix. Extravillous trophoblast cells, which may also exert phagocytic activities, showed no intracellular immunoreactivity for MPO or HOCl-modified proteins. The present findings indicate that the generation of $\mathrm{HOCl}$-modified proteins during normal pregnancy is a physiologic rather than a pathophysiologic process. (Lab Invest 2001, 81:543-554).
\end{abstract}

$T$ he human placenta is hemochorial, meaning that it is directly bathed in maternal blood. One of the prime functions of the placenta is the transportation of substrates for energy metabolism as well as precursors for biosynthesis of macromolecules needed during the period of growth and development in utero (Dockery et al, 2000; Watson and Burton, 1998). Exchange between the maternal and fetal circulation occurs in the placenta through chorionic villi. These structures are composed of an outer layer of syncytiotrophoblast, which is maintained by the underlying mononuclear cytotrophoblast cells. Subjacent to the trophoblast layer is a well-developed basal lamina, which separates the trophoblast compartment from the mesenchymal core. The core is composed of fibroblasts, placental macrophages (Hofbauer cells), and cells of the placental vasculature, all of which are embedded in an extensive extracellular matrix. Some

\section{Received December 14, 2000.}

This work was supported by the Austrian National Bank Grant OENB 8778 (to EM and AH) and by Grants P14109-GEN (to WS) and P14186-MED (to EM) from the Austrian Research Foundation (FWF). This paper was presented in part at the 5 th Conference of the International Federation of Placenta Associations, the 8th Meeting of the European Placenta Group, Schladming, Austria, September 1999.

Address reprint requests to: Dr. Ernst Malle, Karl-Franzens University Graz, Institute of Medical Biochemistry, Harrachgasse 21, A-8010 Graz, Austria/Europe. E-mail: ernst.malle@kfunigraz.ac.at trophoblast cells can be found outside the chorionic villi, ie, the extravillous trophoblast cells (Benirschke and Kaufmann, 1995). These extravillous trophoblast cells deeply invade the uterine wall, a process that is essential for the development of a pregnancy. Interactions of maternal leukocytes and decidual cells with the invading trophoblast cells may play a crucial role in trophoblast migration and invasion, and control the remodeling of the extracellular matrix (Hunt et al, 2000; Loke and King, 2000). Furthermore, this interaction is involved in establishing the immunologic tolerance of the fetus and is mainly regulated by maternal monocytes/macrophages, natural killer cells, and, to a lesser extent, T lymphocytes (Loke and King, 1995).

Monocytes/macrophages, professional phagocytes with the prime function of immunosurveillance and host defence against infection, generate reactive oxygen species (ROS) (Babior, 1978). This reaction, known as the oxidative burst, involves the single electron reduction of oxygen to the superoxide anion radical catalyzed by an NADPH or $\mathrm{NADH}$ oxidase. Superoxide dismutase converts the superoxide anion to $\mathrm{H}_{2} \mathrm{O}_{2}$, which is further converted to water and molecular oxygen by catalase. Under appropriate stoichiometric conditions, the superoxide anion reacts with nitric oxide (a free radical) to form peroxynitrite (Beckman et al, 1994), an oxidant that may cause vascular damage. Two alternative pathways for gen- 

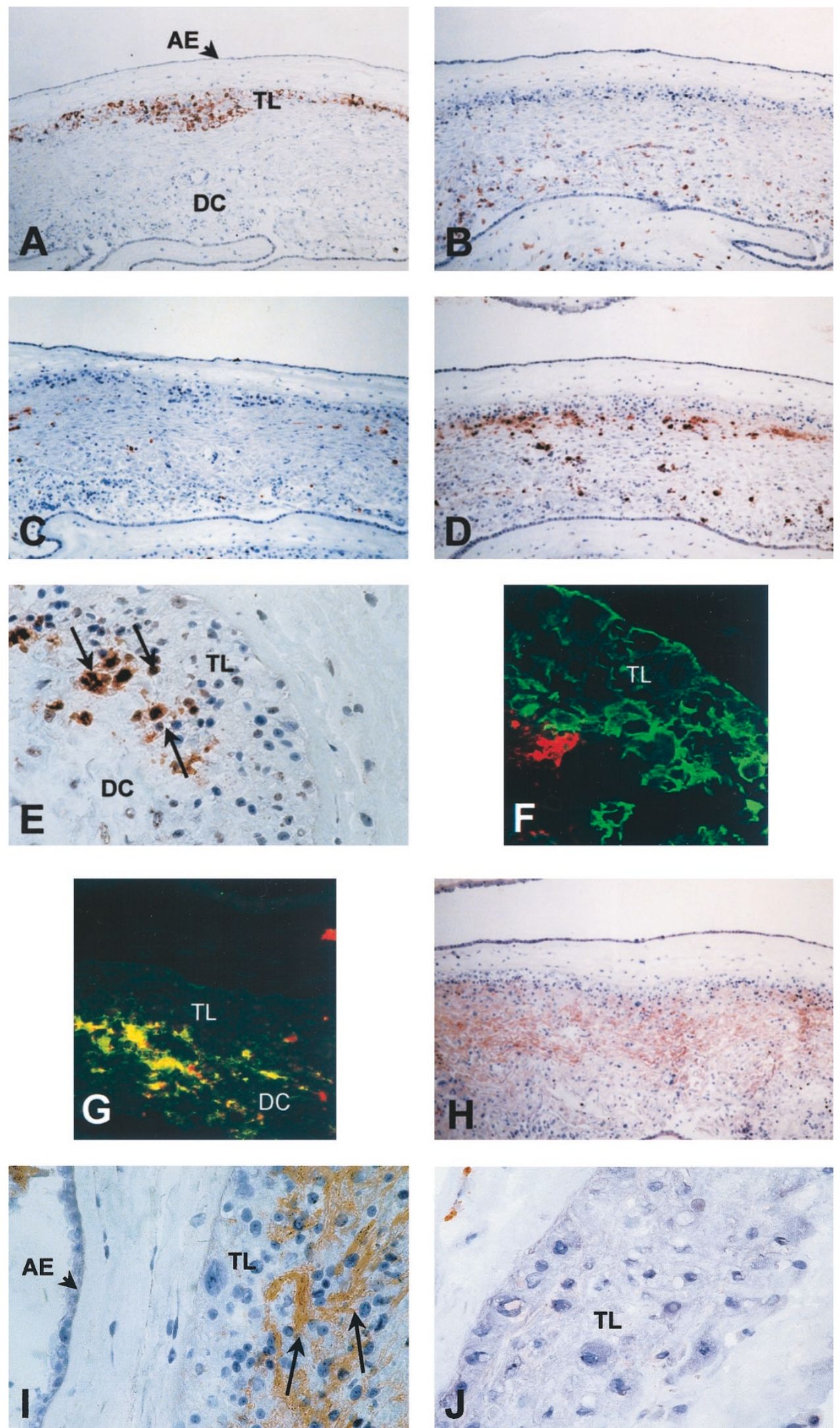

\section{Figure 1.}

Immunohistochemical detection and immunofluorescence double labeling of myeloperoxidase (MPO) and hypochlorous acid (HOCl)-modified proteins in seria sections of fetal membranes. $A$, Identification of trophoblast layer in the chorion, using the cytokeratin marker MNF116 (dilution 1:500) as the primary antibody. $B$, Identification of monocytes/macrophages using the CD163-specific mAb Ber-MAK-3 (dilution 1:50). C, Identification of neutrophils using the elastase-specific mAb M 0752 (dilution 1:50). The same pattern of immunoreactivity was observed using anti-human neutrophil cathepsin $G$ antiserum (not shown). $D$, Immunoreactivity 
erating reactive nitrogen species exist that each involve myeloperoxidase (MPO) (Carr et al, 2000; Hazen et al, 1999). MPO (EC 1.11.1.7) can use nitrite, a major end product of nitric oxide metabolism, as a substrate to nitrate protein tyrosine residues and to initiate lipid peroxidation. Alternatively, MPO may indirectly generate a nitrating intermediate through the secondary oxidation of nitrite with hypochlorous acid/hypochlorite $\left(\mathrm{HOCl} / \mathrm{OCl}^{-}\right)$. $\mathrm{HOCl}$ can be formed only from $\mathrm{H}_{2} \mathrm{O}_{2}$ and chloride ions by MPO (Weiss et al, 1982). This short-lived and diffusible oxidant is believed to have a major role as a potent microbicidal agent in the immune defense. It can participate in a variety of secondary nonenzymic reactions, such as oxidation or chlorination. $\mathrm{HOCl}$ reacts with thiols and thioesters, Fe-S centers, nucleotides, with unsaturated fatty acids to form chlorohydrins, and with amines to form reactive chloramines, which are powerful oxidants (Thomas et al, 1986). The presence of HOCl-modified target compounds underscores the potential role of the $\mathrm{MPO}-\mathrm{H}_{2} \mathrm{O}_{2}$-chloride pathway during early and acute stages of inflammation, eg, atherosclerosis (Malle et al, 2000) and glomerular and tubulointerstitial injury (Malle et al, 1997). The predominant in vivo sources for MPO released during the oxidative burst are neutrophils and monocytes, in which MPO makes up $5 \%$ and $1 \%$ in the total cell protein content, respectively.

The course of pregnancy is accompanied by a balance of generation and decomposition of ROS, which may simply derive from oxygen consumption by placental tissue and active energy metabolism from mitochondrial activities. Both changes in the levels of lipid peroxides during pregnancy (Takehara et al, 1990; Walsh et al, 2000) and immunohistochemical localization of epitopes indicative of lipid peroxidation (Casasco et al, 1997) and nitrating intermediates (Myatt et al, 1996), suggest that ROS are generated in human placenta during normal gestation. In a recent in vitro study, $\mathrm{HOCl}$-exposure of human amnio-chorion led to changes in the cellular characteristics of amnionic fibroblast and cytotrophoblast cells and changes in the extracellular matrix (Plessinger et al, 2000). As MPO is believed to be an essential enzyme in generating modified/oxidized proteins, the purpose of the present study was to localize MPO in human placental tissues and to identify the cellular source of MPO. We also identified and localized HOCl-modified proteins in term placental tissues during normal gestation.

\section{Results}

\section{Immunohistochemistry}

Fetal Membranes. The fetal membranes arise from three different tissues: (1) the amnion, derived from fetal ectoderm, which consists of the amnionic epithelium, underlined by a basal lamina, and the amnionic connective tissue; (2) the chorion laeve, containing fetally derived trophoblast cells and fetal mesenchyme; and (3) the decidua capsularis, which is the outermost layer of the fetal membranes and represents maternal decidual tissue. As fetally derived trophoblast cells are only present in the chorionic part of the fetal membranes, a trophoblast marker (MNF116) was used to localize the chorion laeve and to identify trophoblast cells located within this area. Trophoblast cells were present in the central layer of the fetal membranes (Fig. 1A). Tissue macrophages and activated monocytes, recognized by their immunoreactivity to the mAb Ber-MAK-3, were regularly found in the connective tissue of the amnion, the chorion laeve, and the decidua capsularis (Fig. 1B). Neutrophils, identified by antibodies raised against neutrophil-derived elastase and cathepsin G, were present in the chorion laeve and the decidua capsularis (Fig. 1C). Immunoreactivity for MPO was located in the decidua capsularis and the chorion laeve but not in the amnion (Figure 1D). Immunoreactivity for MPO was associated with neutrophils and monocytes/macrophages but was also present in the extracellular matrix. Pronounced MPO immunoreactivity was found at the feto-maternal contact zone (Fig. 1E), where fetal trophoblast cells (devoid of MPO immunoreactivity) come close to maternal cells of the decidua capsularis. However, the connective tissue of the amnion was devoid of MPO immunoreactivity.

An immunofluorescence double-labeling technique was used to determine whether trophoblast cells or leukocytes were the in vivo source for MPO. Figure $1 \mathrm{~F}$ shows that trophoblast cells (identified with MNF116) were devoid of MPO, both on the cellular surface and in the cytoplasm. However, pronounced MPO immunoreactivity was usually colocalized with CD45immunoreactive cells (Fig. 1G).

A pattern of immunoreactivity similar to the one shown for MPO (Fig. 1D and E) was obtained with $\mathrm{mAb}$ (clones 2D10G9 and 10A7H9) that specifically recognize $\mathrm{HOCl}$-modified proteins (Fig. $1 \mathrm{H}$ ). Immunoreactivity for $\mathrm{HOCl}$-modified proteins was not present intracellularly but was cell-associated and also lo-

\footnotetext{
for MPO using polyclonal rabbit anti-human MPO IgG (dilution 1:1000). E, An enlargement of $(D)$ demonstrates the presence of MPO (arrows) at the borderline between chorion and decidua capsularis. F, Double labeling for trophoblast cells and MPO: MNF116-FITC (dilution 1:25, green) and rabbit anti-human MPO IgG (dilution 1:1000) were used as primary antibodies. Cy-3-labeled anti-rabbit IgG (dilution 1:500, red) was used as a secondary antibody. G, Double labeling for leukocytes and MPO: phycoerythrin(PE)-labeled anti-CD45 (dilution 1:40, red) and rabbit anti-human MPO IgG (dilution 1:1000) were used as primary antibodies. Cy-2-labeled anti-rabbit IgG (dilution 1:500, green signal) was used as a secondary antibody. $\mathrm{H}$, Immunoreactivity for HOCl-modified proteins in the same area shown in $A$ to $D$, using mAb 2D10G9 (dilution 1:1). The same pattern of immunoreactivity was observed using mAb 10A7H9 as the primary antibody at the same dilution (not shown). I, An enlargement of $(H)$ demonstrates the presence of HOCl-modified proteins in the extracellular matrix between trophoblast cells (arrows). The same pattern of immunoreactivity was observed using mAb $10 \mathrm{~A} 7 \mathrm{H} 9$ as a primary antibody at the same dilution (not shown). J, Negative control of $H$ and $I$ : Preabsorption of mAb 2D10G9 with a 30-fold molar excess of HOCI-LDL. TL, layer of trophoblast cells; $A E$, amnion epithelium; $D C$, decidua capsularis. Original magnification, $A$ to $D$ and $H, \times 100 ; E$ and $I, \times 400 ; J, \times 630$. Scan field areas (x/y dimension), $F=100 \mu \mathrm{m} ; G=200 \mu \mathrm{m}$.
} 

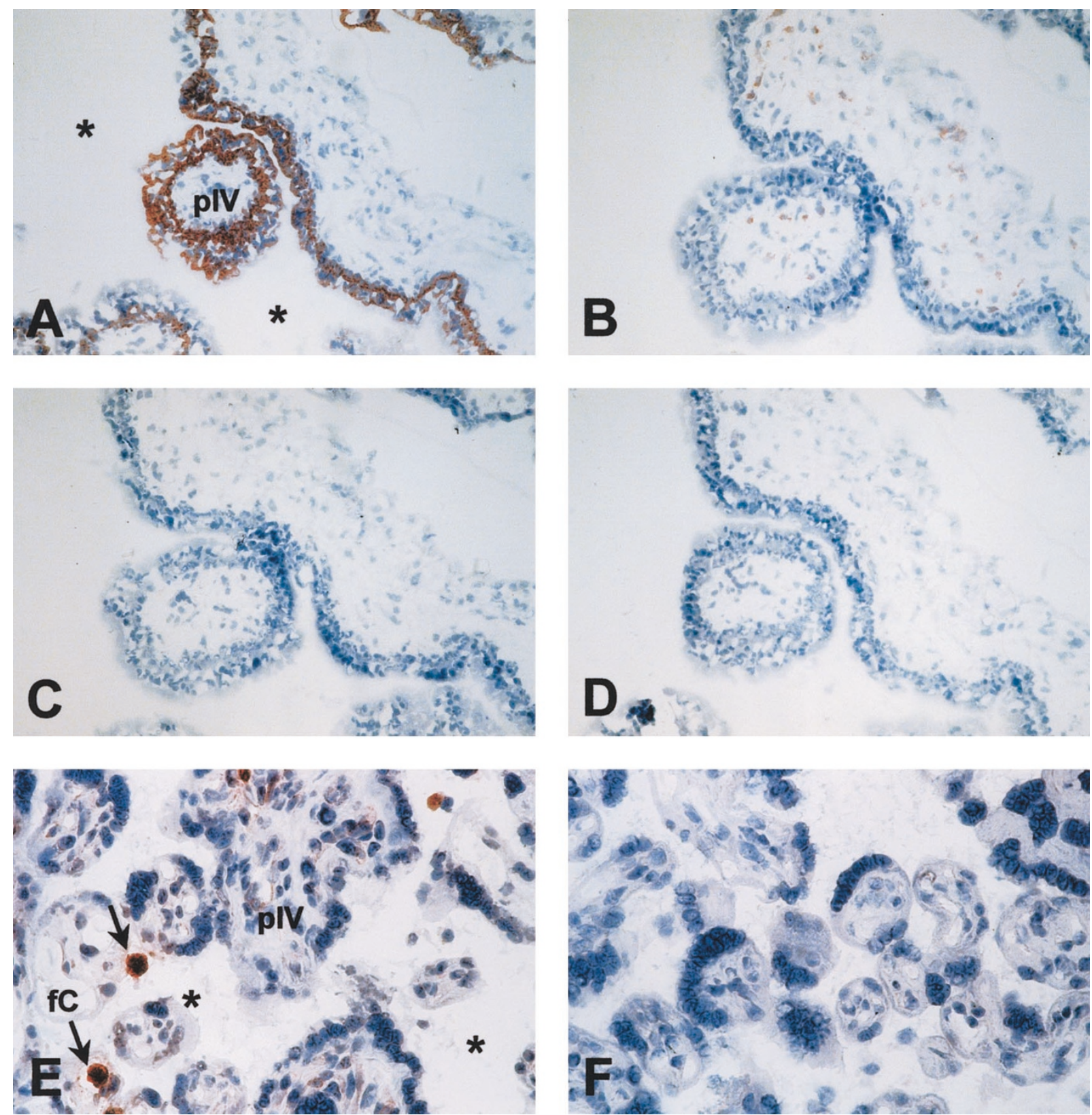

Figure 2.

Immunohistochemical detection of MPO and HOCl-modified proteins in serial sections of first trimester $(A$ to $D)$ and term placental villi $(E$ and $F)$. $A$, Identification of trophoblast cells using the cytokeratin marker MNF116 (dilution 1:500) as the primary antibody. B, Identification of fetal macrophages (Hofbauer cells) using the mAb Ber-MAK-3 (dilution 1:50). C, Immunoreactivity for MPO using polyclonal rabbit anti-human MPO IgG (dilution 1:1000). $D$, Immunoreactivity for HOCI-modified proteins in the same area shown in $A$ to $C$, using mAb 2D10G9 (dilution 1:1) as a primary antibody. $E$, Immunoreactivity for MP0 using polyclonal rabbit anti-human MPO IgG (dilution 1:1000). The arrows indicate MPO immunoreactivity in maternal and fetal blood cells. $F$, Immunoreactivity for HOCl-modified proteins in the same area shown in $(E)$ using 2D10G9 (dilution 1:1) as the primary antibody. p/V, placental villi; $f C$, fetal capillary; *, intervillous space containing maternal blood. Original magnification, $A$ to $F, \times 400$.

cated extracellularly along fibrous structures, eg, the matrix between the trophoblast cells of the chorion laeve (Fig. 11). No immunoreactivity was observed after preabsorption of mAb 2D10G9 (or 10A7H9) with a 30 -fold molar excess of $\mathrm{HOCl}$-modified low-density lipoprotein (HOCl-LDL) (Fig. 1J), or when the specific primary antibodies were replaced by mousenonimmune serum (containing various IgGsubclasses) or mouse $\operatorname{lgG}_{1}$ (data not shown).

Villous Part of the Placenta. The fetally derived placental villi, protruding into the intervillous space, are composed of a villous core that contains fetal blood vessels, fetal macrophages (ie, Hofbauer cells), and fibroblasts, embedded in connective tissue. The villi are covered by syncytiotrophoblast and thus directly bathed in maternal placental blood. Both villous cytotrophoblast cells underlying the syncytiotrophoblast and the syncytiotrophoblast were immunoreactive for the cytokeratin marker MNF116 (Fig. 2A). Hofbauer cells were localized within this tissue (Fig. $2 B$ ), whereas immunoreactivity for neutrophil-derived elastase (not shown) was not observed. Immunoreac- 
tivity for MPO (Fig. 2C) and $\mathrm{HOCl}$-modified proteins (Fig. 2D) was absent in placental villi from the first trimester of pregnancy, during Weeks 7 through 12 of gestation. At term of gestation, pronounced immunoreactivity for MPO (Fig. 2E), but not for $\mathrm{HOCl}$-modified proteins (Fig. 2F), was apparent in both maternal (located in the intervillous space) and fetal (within the villous blood vessels) blood cells, eg, neutrophils and monocytes. In some preparations, an additional faint immunoreactivity for MPO was associated with endothelial cells of the fetal blood vessels (Fig. 2E).

Decidua Basalis. The uterine decidua basalis (Fig. 3), a composite tissue that is part maternal and part fetal in origin, lies beneath the villous placenta. It consists of invading trophoblast cells (Fig. 3A), interposed between maternal cells, decidual stromal cells, epithelial cells of uterine glands, leukocytes (which may account for up $40 \%$ of total maternal cells (Loke and King, 1995)), and other cells of maternal blood vessels. The specific trophoblast marker MNF116 was used to differentiate between the trophoblast-invaded decidua basalis, which takes part in the formation of the later basal plate, and the non trophoblast-invaded decidua parietalis. In the same area (shown in Fig. 3A), monocytes/macrophages were identified with the mAb Ber-MAK-3 (Fig. 3B), whereas labeling for neutrophils, using antibodies against neutrophil-derived elastase (Fig. 3C) or cathepsin G (not shown), was very rare. MPO immunoreactivity was associated with monocytes/macrophages and was also located in the extracellular matrix (Fig. 3D).

As shown in fetal membranes, the immunofluorescence double-labeling technique revealed that trophoblast cells (identified with MNF116) were not immunoreactive for human MPO IgG (Fig. 3E). Colocalization of MPO was only observed with cells immunoreactive for CD45, ie, leukocytes (Fig. 3F).

$\mathrm{HOCl}-$ modified proteins were not identified in the decidua basalis during the first trimester of pregnancy (Weeks 7 to 12) (Fig. 3G). Immunoreactivity was also absent when mouse-nonimmune $\lg G$ was used as a primary antibody (Fig. 3H).

Basal Plate. The basal plate is that part of the materno-fetal junctional zone that adheres to the delivered placenta. It is made up of an admixture of trophoblast cells, various endometrial stromal cells, decidual cells, uteroplacental vessels, and residues of endometrial glands without strict order. All of these tissue components are embedded in ample fibrinoid (Lang et al, 1994), which makes up the glossy to fibrillar ground substance. Trophoblast cells readily infiltrated the basal plate of a term placenta (Fig. 4A). Additionally, immunoreactivity for MPO was associated with deposits that may have been derived from monocytes or remnants of other cells but not from trophoblast cells (Fig. 4B). In contrast to the decidua basalis (Fig. 3G), pronounced and heterogeneously distributed immunoreactivity for $\mathrm{HOCl}$-modified proteins was present in the basal plate from term placenta (Fig. 4C). Similar to the chorion laeve (Fig. $1 \mathrm{H}$ and I), immunoreactivity for $\mathrm{HOCl}$-modified epitopes was not present intracellularly, but was associated with the extracellular matrix between trophoblast cells (Fig. 4C). Preabsorption of mAb 2D10G9 with HOCl-LDL (Fig. 4D), but not with native LDL (not shown), prevented antibody binding, demonstrating that the immunoreactivity was specific for $\mathrm{HOCl}$-modified proteins.

Table 1 summarizes all of the immunohistochemical results on the presence of MPO and $\mathrm{HOCl}$-modified proteins in human placental tissues.

\section{Immunoblotting Experiments}

Identification of MPO and HOCl-Modified Epitopes. To strengthen the immunohistochemical findings on MPO and $\mathrm{HOCl}$-modified proteins, frozen tissue sample homogenates, the same as shown in Figure 1, were used for Western blot analysis. The rabbit antibody to human MPO recognized the heavy subunit of MPO in detergent extracts of human neutrophils (Fig. $5 A$, lane 1) and placental tissues (Fig. 5A, lane 3). The $58 \mathrm{kd}$ MPO band comigrated with authentic MPO (Fig. $5 A$, lane 2). Omission of anti-human MPO IgG (not shown) or preabsorption of the primary antibody with purified MPO (Fig. 5A, lane 4) did not reveal any immunoreactivity. In most tissue samples, a diffuse smear indicated the presence of $\mathrm{HOCl}$-modified proteins in placental tissues (Fig. 5B, lane 1). The heterogeneity in immunoreactive bands was probably caused by cross-linking of $\mathrm{HOCl}$-modified proteins (ie, chloramines); which agrees with our previous findings in kidney tissues (Malle et al, 1997; Scheuer et al, 2000). Again, omission of the primary antibody (not shown) or replacement of $\mathrm{mAb}$ with an $\lg \mathrm{G}$ isotype control (not shown) eliminated all immunoreactivity. Competition experiments with both mAb (clone 2D10G9 and 10A7H9) preabsorbed with $\mathrm{HOCl}-$ modified human serum albumin ( $\mathrm{HOCl}-\mathrm{HSA}$ ) (Fig. 5B, lane 2) or $\mathrm{HOCl}-\mathrm{LDL}$ (not shown) at a molar ratio of 1:30 prevented antibody binding, demonstrating that the immunoreactivity was specific for $\mathrm{HOCl}$-modified proteins rather than a nonspecific effect.

\section{Discussion}

MPO, an estrogen-inducible peroxidase, is present in placental tissues (Lyttle and DeSombre, 1977) but its localization within these was not known. In situ identification performed in the present study revealed immunoreactive MPO in fetal membranes and the basal plate and in maternal and fetal blood cells. Immunoreactivity for MPO in term placental tissues was mostly associated with cell remnants and monocytes. Although we did not differentiate between macrophages and monocytes, the latter cell type seemed to be the predominant source for immunoreactive MPO; this granule peroxidase is generally lost as monocytes mature into macrophages (Koeffler et al, 1985). This might explain the absence of MPO immunoreactivity in macrophages of villous tissue at all stages of pregnancy. However, MPO secreted during the oxidative burst of monocytes can be taken up by macrophages (Shepherd and Hoidal, 1990), which in 

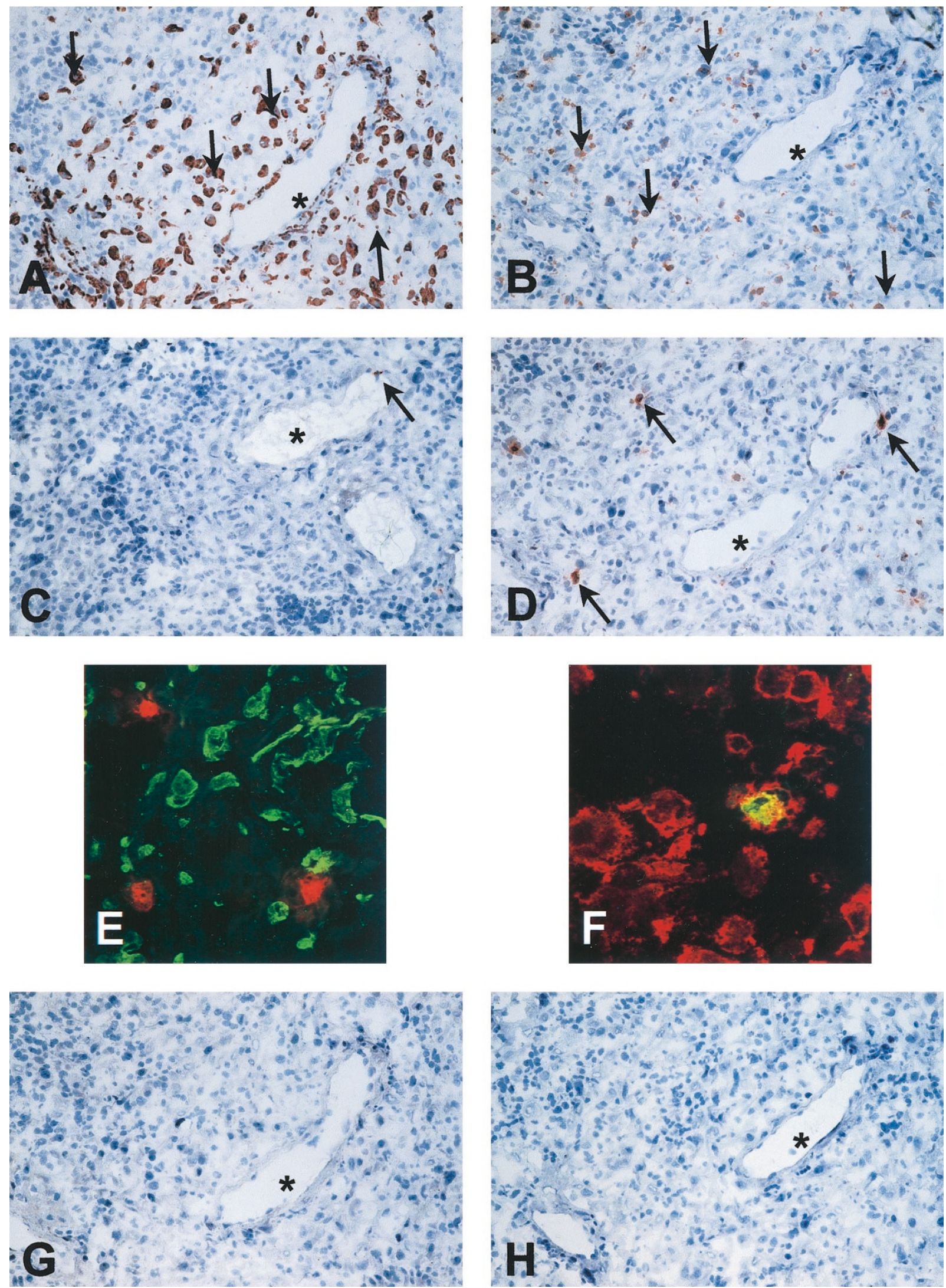

Figure 3.

Immunohistochemical detection and immunofluorescence double labeling of MPO and HOCl-modified proteins in serial sections of the decidua basalis. $A$ Identification of extravillous trophoblast cells (arrows) within the maternal decidua basalis using mAb MNF116 (dilution 1:500) as the primary antibody. $B$, Identification of maternal monocytes/macrophages (arrows) using mAb Ber-MAK-3 (dilution 1:50). C, Identification of neutrophils (arrow) using the elastase-specific 

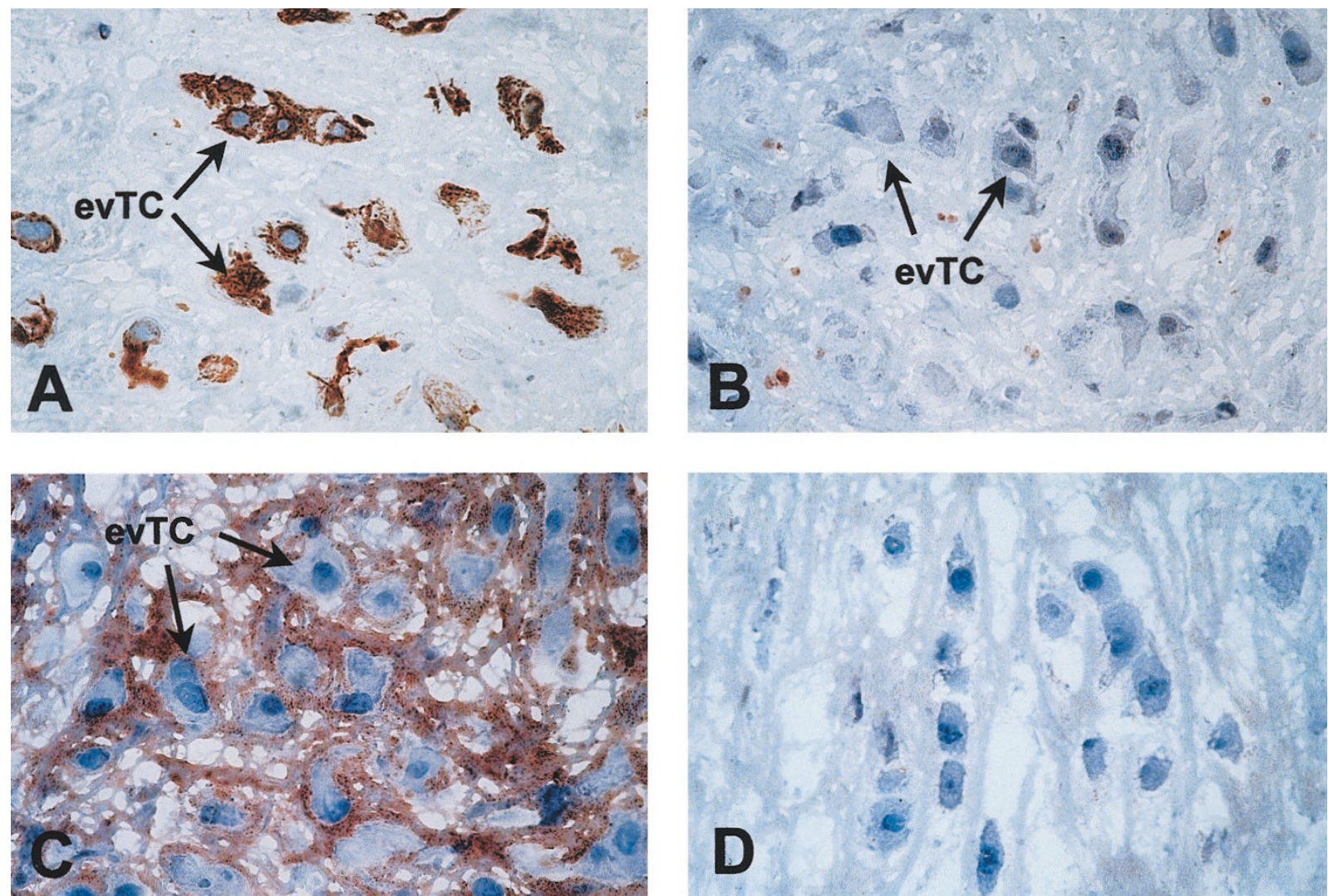

\section{Figure 4.}

Immunohistochemical detection of MPO and $\mathrm{HOCl}$-modified proteins in serial sections of a term placenta basal plate. A, Identification of extravillous trophoblast cells within the basal plate using mAb MNF116 (dilution 1:500) as the primary antibody. B, Immunoreactivity for MP0 using polyclonal rabbit anti-human MPO IgG (dilution 1:1000). $C$, Immunoreactivity for $\mathrm{HOCl}$-modified proteins in the same area shown in $A$ and $B$ using mAb $2 \mathrm{D} 10 \mathrm{G9}$ (dilution 1:1) as a primary antibody. $D$, Negative control of $C$ : preabsorption of 2D10G9 with a 30 -fold molar excess of HOCl-LDL. EvTC, extravillous trophoblast cells. Original magnification, $A$ to $D$, $\times 400$.

turn may enhance the macrophages capacity for phagocytosis and intracellular killing of microorganisms (Lefkowitz et al, 1996). HOCl-modified proteins could only be detected in term placenta, not during the first trimester of pregnancy until week 12 . This is irrespective of the fact that during Weeks 8 to 13 an increased generation of ROS could result from enhanced placental and endometrial partial pressure of oxygen (Rodesch et al, 1992). However, as tissues between week 12 and term were not available, the onset of immunoreactivity for $\mathrm{HOCl}$-modified proteins is unknown. $\mathrm{HOCl}$-modified epitopes are only present in areas formed by fetally derived tissue or maternal decidual tissue (basal plate and fetal membranes); areas where fetal extravillous trophoblast cells invade the maternal decidua and may trigger the maternal immune system (Loke and King, 1995). HOCl-modified proteins were not found intracellularly but were cell associated or found along fibrous structures of the extracellular matrix, similar to other tissues (Malle et al, 1997, 2000).

A high rate of infiltration of monocytes/macrophages over a longer period reflects a chronic inflammation, which leads to pathologic alterations. During normal host defense, neutrophils are the major in vivo source for MPO. However, because of the absence of neutrophils in human placental tissues, in vivo generation of $\mathrm{HOCl}$ must be predominantly mediated by the MPO- $\mathrm{H}_{2} \mathrm{O}_{2}$-chloride system of activated monocytes, similar to findings in human atherosclerotic lesions. There, monocyte-derived MPO (Daugherty et al, 1994) seems to be the major source for $\mathrm{HOCl}$ and $\mathrm{HOCl}-$ modified (lipo)proteins (Hazell et al, 1996; Malle et al, 2000). Taurine, the most important in vivo scavenger for $\mathrm{HOCl}$, significantly impairs lesion severity in dietinduced hypercholesterolemia (Petty et al, 1990), supporting the notion that $\mathrm{HOCl}$ and the subsequent

mAb M 0752 (dilution 1:50). The same pattern of immunoreactivity was observed using anti-human neutrophil cathepsin $\mathrm{G}$ antiserum (not shown). $D$, Immunoreactivity for MPO (arrows) using polyclonal rabbit anti-human MPO IgG (dilution 1:1000). E, Double labeling for trophoblast cells and MPO: MNF116-FITC (dilution 1:25, green) and rabbit anti-human MPO IgG (dilution 1:1000) were used as primary antibodies. Cy-3-labeled anti-rabbit IgG (dilution 1:500, red) was used as a secondary antibody. F, Double labeling for leukocytes and MPO: PE-labeled anti-CD45 (dilution 1:40, red) and rabbit anti-human MPO IgG (dilution 1:1000) were used as primary antibodies. Cy-2-labeled anti-rabbit IgG (dilution 1:500, green) was used as a secondary antibody. G, Immunoreactivity for HOCl-modified proteins in the same area shown in $A$ to $D$, using mAb 2D10G9 (dilution 1:1) as the primary antibody. $H$, Negative control of $D$ : rabbit nonimmune serum was used as the primary antibody. ${ }^{*}$ maternal blood vessels. Original magnification, $A$ to $D, G$ and $H, \times 200$. Scan field areas (x/y dimension), $E, F=100 \mu \mathrm{m}$. 
Table 1. Immunohistochemical Localization of MPO and HOCI-Modified Proteins in Human Placental Tissues

\begin{tabular}{|c|c|c|c|}
\hline & Cell type & MPO & HOCl-epitopes \\
\hline \multirow[t]{5}{*}{ Fetal membranes } & Extravillous cytotrophoblast cell & - & a \\
\hline & Fibroblasts & - & a \\
\hline & Monocytes/macrophages & + & a \\
\hline & Neutrophils & + & $\mathrm{a}$ \\
\hline & Extracellular matrix & + & + \\
\hline \multirow{7}{*}{$\begin{array}{l}\text { Placental villi } \\
\qquad(1 \text { st trimester }+ \text { term) }\end{array}$} & Hofbauer cells & - & - \\
\hline & Endothelial cells & $-1+$ & - \\
\hline & Stromal cells & - & - \\
\hline & Syncytiotrophoblast & - & - \\
\hline & Villous cytotrophoblast cells & - & - \\
\hline & Maternal blood cells & + & - \\
\hline & Fetal blood cells & + & - \\
\hline \multirow[t]{5}{*}{ Decidua } & Decidual cells & - & - \\
\hline & Monocytes/macrophages & + & - \\
\hline & Endothelial cells & - & - \\
\hline & Extravillous cytotrophoblast cell & - & - \\
\hline & Extracellular matrix & - & - \\
\hline \multirow[t]{5}{*}{ Basal plate } & Decidual cells & - & - \\
\hline & Monocytes/macrophages & + & - \\
\hline & Endothelial cells & - & - \\
\hline & Extravillous cytotrophoblast cell & - & $\mathrm{a}$ \\
\hline & Extracellular matrix & + & + \\
\hline
\end{tabular}

- , no staining; $-/+$, weak staining; +, strong staining; a, cell-associated but not intracellularly.

formation of chloramines account for deleterious effects during inflammatory conditions.

The oxidation of proteins by $\mathrm{HOCl}$ is also believed to be a leading event in glomerular and tubulointerstitial injury (Malle et al, 1997), because enhanced generation of ROS in hyperlipidemia-induced renal damage is tightly coupled with the generation of $\mathrm{HOCl}$-modified proteins (Scheuer et al, 2000). However, in contrast to other inflammatory conditions, such as atherosclerosis and glomerulosclerosis, the accumulation of monocytes/macrophages within the decidua seems to be a part of normal placental development and function. Utero-placental macrophages have the potential to produce a wide range of bioactive mediators, including nitric oxide. Because of regular oxygen and nitrogen species, both 4-hydroxynonenal (a lipid peroxidation product) and nitrotyrosine (an in vivo marker for peroxynitrite), are present in normal human placental tissues (Casasco et al, 1997; Many et al, 2000; Nakatsuka et al, 1999). These findings support the contention that oxidative modification is a normal physiologic process in the placenta. However, it is probable that enhanced oxidative stress during inflammation is a leading cause for increased generation of modified proteins in placental tissues under pathologic conditions, eg, endometriosis, preeclampsia, chorioamnionitis, and diabetes (Kossenjans et al, 2000; Murphy et al, 1998; Myatt et al, 1996; Nakatsuka et al, 1999).

The antibodies used to identify $\mathrm{HOCl}$-modified proteins in human placental tissues react with $\mathrm{HOCl}-$ modified epitopes generated by $\mathrm{HOCl}$ added as reagent or generated by the $\mathrm{MPO}-\mathrm{H}_{2} \mathrm{O}_{2}$-chloride pathway (Malle et al, 2000). The antibodies do not cross-react with other epitopes generated by lipid peroxidation or nitrating processes. Our findings do not exclude the colocalization of epitopes generated by lipid peroxidation or nitration with MPO-generated oxidation products; (i) tyrosyl radicals, generated by MPO in vivo in the absence of chloride ions, may initiate lipid peroxidation (Savenkova et al, 1994) and (ii) secondary radicals of protein-associated chloramines could also lead to the modification of proteins by secondary lipid peroxidation products (Hazell et al, 1999). This assumption is supported by the faint immunoreactivity for nitrating intermediates associated with most cytotrophoblast cells within the uterine wall in normal term pregnancy but not in the first trimester of pregnancy (Many et al, 2000). The epitopes generated by the MPO- $\mathrm{H}_{2} \mathrm{O}_{2}$-chloride pathway are primarily protein-chloramine adducts, interesting oxidants that could act as bioactive molecules by linking inflammation and immune response (Marcinkiewicz, 1997). Early in acute inflammation, $\mathrm{HOCl}$ and other reactive and cytotoxic chlorine-derived oxidants may contribute to the neutrophil-dependent inflammatory reaction. When the acute phase continues and the number of macrophages increases, as occurs during invasion of trophoblast cells, chlorinated antigens may be taken up by local antigenpresenting cells, contributing to enhanced activation of antigen specific T cells. In support of these findings, $\mathrm{HOCl}-\mathrm{LDL}$ was reported to induce synthesis of monocyte-derived IL-8 (Woenckhaus et al, 1998), a cytokine with chemotactic activities for $\mathrm{T}$ cells. There is evidence that IL-8 is released from the amnion, decidua, and the placenta at term (Laham et al, 1999; Osmers et al, 1995). A further role for chloramines 

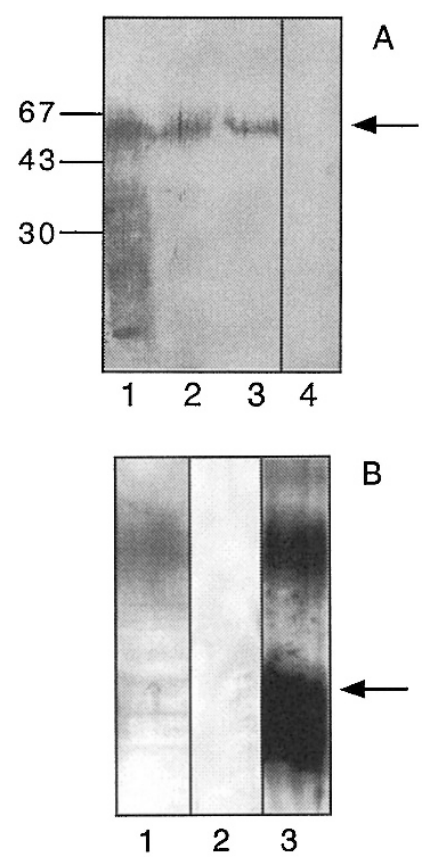

Figure 5.

Immunoblot of MPO $(A)$ and $\mathrm{HOCl}$-modified proteins $(B)$ in placental tissues. Antigens were separated by SDS-PAGE on $5 \%$ to $15 \%$ gradient polyacrylamide gels under reducing conditions and transferred to nitrocellulose. MPO was detected using rabbit anti-human MPO IgG and visualized with alkalinephosphatase conjugated goat anti-rabbit IgG. HOCl-modified proteins were detected using mAb 2D10G9 and visualized with peroxidase-conjugated goat anti-mouse IgG using the ECL Western blotting system. Antigens used: $A$, lane 1, positive control: detergent extracts of human neutrophils $\left(1 \times 10^{6}\right.$ cells $) ; A$, lane 2, positive control: purified human MP0 standard ( $0.1 \mu \mathrm{g} / \mathrm{lane}) ; A$, lane 3 , detergent protein fraction of chorion laeve isolated from human placental tissues; $A$, lane 4, negative control of lane 3: the rabbit anti-human MPO IgG was preabsorbed with purified MPO (at a molar ratio of 35:1) for 2 hours at $22^{\circ} \mathrm{C}$. Molecular masses are given in $\mathrm{kd}$. $B$, lane 1 , detergent protein fraction (60 $\mu \mathrm{g}$ protein/lane) of chorion laeve isolated from human placental tissues; $B$, lane 2, negative control of lane 1 : the primary antibody was preabsorbed with a 30-fold molar excess of HOCl-HSA; B, lane 3, positive control: HOCl-HSA (5 $\mu \mathrm{g} / \mathrm{lane})$. The arrow indicates the position of the $67 \mathrm{kd}$ HOCl-HSA monomer.

during immunomodulation could involve the elimination of activated Fas-receptor-expressing leukocytes, because chloramines may enhance Fas-induced apoptosis in the lymphoblastic T-cell line Jurkat (Ogino et al, 2000). A similar process has been recently addressed in endothelial cells, where $\mathrm{Cu}^{2+}$-oxidizedLDL, a convenient experimental model of physiologically important LDL modification, activated Fasreceptor-mediated apoptosis (Sata and Walsh, 1998).

In summary, different oxidative pathways may affect the generation of ROS, the subsequent formation of modified proteins in placental tissues, and their relative contributions during gestation. Our results show that the MPO- $\mathrm{H}_{2} \mathrm{O}_{2}$-chloride system leads to the formation of $\mathrm{HOCl}$-modified products, which are present in normal human term placenta but not during Weeks 7 to 12 of the first trimester of pregnancy. We therefore propose that oxidative modification of proteins, ie, the presence of $\mathrm{HOCl}$-modified proteins, is a normal physiologic process in the human placenta during normal pregnancy. The presence of $\mathrm{HOCl}$-modified epitopes is restricted to areas of the placenta where interaction between fetal trophoblast cells and maternal tissues takes place, indicating local stimulation of the maternal immune system. Whether chloramines provide a bridge between inflammation and the immune system and between the afferent branches of the innate and acquired immune systems (Marcinkiewicz, 1997) also in the placenta deserves further investigation.

\section{Material and Methods}

\section{Antibodies}

The following murine $m A b$ (subtype: $\lg _{1}$ ) were purchased from DAKO (Vienna, Austria) and used as primary antibodies: MNF116- and MNF116fluorescein isothiocyanate (FITC), specific for cytokeratins present in all trophoblast populations (Blaschitz et al, 2000); Ber-MAK-3, specific for CD163 present on tissue macrophages and activated monocytes (Blaschitz et al, 2000); and $M$ 0752, specific for human neutrophil elastase. The $\operatorname{lgG}_{2 b_{\kappa}}$ mAb 2D10G9 and 10A7H9 were raised against HOCl-LDL (800 molecules of $\mathrm{HOCl}$ per LDL particle) (Malle et al, 1995; Malle et al, 2000). Both $\mathrm{mAb}$ are highly specific for $\mathrm{HOCl}-\mathrm{LDL}$ and other $\mathrm{HOCl}$-modified proteins/epitopes and do not cross-react with native LDL or other modified/oxidized LDL preparations such as peroxynitriteLDL, hemin-LDL, $\mathrm{Cu}^{2+}$-oxidized-LDL, 4-hydroxynonenal-LDL, malondialdehyde-LDL, glycated-LDL, or acetylated-LDL. The $\operatorname{lgG}_{1} \mathrm{mAb} K C 56$, specific for the leukocyte common antigen CD45, and labeled with phycoerythrin (PE) was obtained from Coulter Instrumentation (Vienna, Austria).

The following polyclonal rabbit antibodies obtained from DAKO were used as primary antibodies: antihuman MPO lgG fraction and anti-human neutrophil cathepsin $\mathrm{G}$ antiserum.

The following polyclonal goat antibodies, obtained from Jackson Immunoresearch (West Grove, Pennsylvania) were used as detection antibodies in immunofluorescence double labeling: anti-rabbit IgGcyanine(Cy)-2 and anti-rabbit IgG-Cy-3.

\section{Tissues}

Human term placenta was obtained after normal vaginal delivery. First-trimester placental and decidual tissue from pregnancies in the period between Weeks 7 and 12 of gestation was obtained after termination of pregnancy by curettage for psychosocial reasons. This procedure was approved by the Ethical Committee of the Medical School, Karl-Franzens-University, Graz, Austria.

Immunohistochemical studies were performed on frozen sections of placental and decidual tissues from different normal pregnancies $(n=7)$. Tissue samples were either snap frozen in liquid nitrogen for later processing or immediately frozen in a cryostat (Microm HM 500 OM; Microm, Walldorf, Germany) supported by tissue freezing medium (Tissue Tec OCT-compound; Miles, Elkhart, Indiana). Serial cryosections (5 $\mu \mathrm{m})$ were collected on glass slides, air dried for 2 hours at $22^{\circ} \mathrm{C}$, 
fixed in acetone for 5 minutes at $22^{\circ} \mathrm{C}$, and stored at $-40^{\circ} \mathrm{C}$ until required. Western blot experiments were performed on homogenates from fetal membranes (containing either complete membranes or membranes from which the amnion was mechanically removed) or on first trimester or term villous tissue.

\section{Enzyme Immunohistochemistry and Light Microscopy}

Before immunohistochemistry, sections were thawed, fixed once more in acetone for 5 minutes at $22^{\circ} \mathrm{C}$, and rehydrated in PBS ( $\mathrm{pH}$ 7.4) for 10 minutes. Immunohistochemistry was performed using a horseradishperoxidase-labeled streptavidin biotin kit (HRP-LSAB; DAKO), as described (Hammer et al, 1997, 1999). All incubations were performed in a moist chamber at $22^{\circ} \mathrm{C}$, using PBS for washes between the incubation steps.

Briefly, after 5 minutes of incubation with blocking solution, the sections were incubated for 30 minutes with the primary antibodies diluted with antibody diluent (DAKO), followed by sequential 15-minutes incubations with a biotinylated linking antibody (goat anti-mouse or goat anti-rabbit lgG) and peroxidaselabeled streptavidin. The reaction was developed with 3-amino-9-ethyl-carbazole/dimethylformamide (Sigma, Vienna, Austria) and terminated by washing with distilled water. The sections were counterstained with Mayer's hemalum and mounted with Kaiser's glycerol gelatin (Merck, Vienna, Austria).

Control experiments encompassed immunohistochemistry (i) without primary detection antibodies, (ii) with monoclonal or polyclonal nonimmune antibodies as primary antibodies, (iii) without secondary antibodies, or (iv) using $\mathrm{HOCl}$-modified (lipo)proteins as competing antigens (the competitor was preincubated with the primary antibody for 20 minutes at the indicated molar ratios before adding to the section). Pictures were taken with an Axiophot microscope (Zeiss, Oberkochen, Germany).

\section{Double Immunofluorescence and Confocal Laser Scanning Microscopy}

Acetone-fixed cryosections were incubated for 30 minutes with rabbit anti-human MPO IgG, then incubated for 30 minutes with either Cy-2- or Cy-3labeled goat anti-rabbit lgG. The specimens were then incubated with FITC-labeled MNF116 for the detection of trophoblast cells, or with the pan leukocyte marker CD45, labeled with PE, respectively. PBS was used for washing sections between the incubation steps. Sections were mounted with Moviol (CalbiochemNovabiochem, La Jolla, California) and analyzed on a confocal laser scanning microscope (Leica TCS NT; Leica Lasertechnik $\mathrm{GmbH}$, Heidelberg, Germany) equipped with an argon-krypton laser. Signals were detected with a double dichroic beam splitter (488/ $568 \mathrm{~nm}$ ), using a $580 \mathrm{~nm}$ short pass and a $530 \mathrm{~nm}$ band pass filter for Cy-2 or FITC and a $590 \mathrm{~nm}$ long pass filter for $\mathrm{Cy}-3$ or PE. Cy-2 and FITC immunofluorescence were colored green, whereas Cy-3 or PE immunofluorescence were colored red. Colocalization of green and red signals was colored yellow.

\section{Polyacrylamide Gel Electrophoresis and Western Blotting Technique}

Polyacrylamide gel electrophoresis was performed using $3.75 \%$ to $12.5 \%$ polyacrylamide gradient gels with electrophoresis at $150 \mathrm{~V}$ for 90 minutes in a Bio-Rad mini blot chamber (Bio-Rad, Richmond, California) (Malle et al, 2000). Tissue homogenates (fetal membranes, first trimester or term placenta) were dissolved in $1 \mathrm{ml}$ of O'Ferral's buffer (3.04 M glycerol, $0.17 \mathrm{M}$ SDS, $0.14 \mathrm{M}$ Tris/HCl buffer $\mathrm{pH}$ 6.8, containing $5 \%$ mercaptoethanol) and centrifuged at 13,000 rpm for 10 minutes. Aliquots from the supernatant were diluted with sample buffer $(0.1 \mathrm{M}$ Tris $/ \mathrm{HCl} \mathrm{pH} 6.8,4 \%$ SDS, $20 \%$ glycerol) at the ratio of $1: 1(\mathrm{v} / \mathrm{v})$ and incubated at $95^{\circ} \mathrm{C}$ for 5 minutes before application to gels. For Western blotting experiments, proteins were transferred by electrophoresis to nitrocellulose membranes.

$\mathrm{HOCl}$-modified proteins were detected by mAb 2D10G9 or 10A7H9 (dilution 1:25) followed by peroxidase-conjugated rabbit anti-mouse IgG (diluted 1:5000) (Sigma, Taufkirchen, Germany) (Malle et al, 2000). MPO was detected with rabbit polyclonal antihuman IgG (diluted 1:100) followed by alkalinephosphatase conjugated mouse anti-rabbit lgG (diluted 1:5000) (Biomedica, Graz, Austria). To visualize immunoreactive bands, ECL Western-blotting detection reagents and Hyperfilm-ECL (Amersham, Berlin, Germany) were used.

Control experiments encompassed immunoblotting experiments (a) without primary detection antibodies, (b) without secondary antibodies, or (c) using $\mathrm{HOCl}-$ modified (lipo)proteins or MPO as competing antigens (the competitor was preincubated with the primary antibody for 20 minutes at the indicated molar ratios before adding to the nitrocellulose).

\section{Acknowledgements}

The expert technical assistance of Christine Daxböck and Birgit Hirschmugl is appreciated. We are grateful for purified human MPO-samples provided by Dr. Christian Obinger (Institute of Chemistry, University of Agricultural Sciences, Vienna, Austria).

\section{References}

Babior BM (1978). Oxygen-dependent microbial killing by phagocytes. N Engl J Med 298:659-668.

Beckman JS, Chen J, Ischiropoulos H, and Crow JP (1994). Oxidative chemistry of peroxynitrite. Methods Enzymol 233: 229-240.

Benirschke K and Kaufmann P (1995). Pathology of the human placenta. New York: Springer Verlag.

Blaschitz A, Weiss U, Dohr G, and Desoye G (2000). Antibody reaction patterns in first trimester placenta: Implications for trophoblast isolation and purity screening. Placenta 21:733741. 
Carr AC, McCall MR, and Frei B (2000). Oxidation of LDL by myeloperoxidase and reactive nitrogen species: Reaction pathways and antioxidant protection. Arterioscler Thromb Vasc Biol 20:1716-1723.

Casasco A, Calligaro A, Casasco M, Tateo S, Icaro Cornaglia A, Reguzzoni M, and Farina A (1997). Immunohistochemical localization of lipoperoxidation products in normal human placenta. Placenta 18:249-253.

Daugherty A, Dunn JL, Rateri DL, and Heinecke JW (1994). Myeloperoxidase, a catalyst for lipoprotein oxidation, is expressed in human atherosclerotic lesions. J Clin Invest 94: 437-444.

Dockery P, Bermingham J, and Jenkins D (2000). Structurefunction relations in the human placenta. Biochem Soc Trans 28:202-208.

Hammer A, Blaschitz A, Daxbock C, Walcher W, and Dohr G (1999). Fas and Fas-ligand are expressed in the uteroplacental unit of first-trimester pregnancy. Am J Reprod Immunol $41: 41-51$.

Hammer A, Hutter H, Blaschitz A, Mahnert W, Hartmann M, Uchanska-Ziegler B, Ziegler A, and Dohr G (1997). Amnion epithelial cells, in contrast to trophoblast cells, express all classical HLA class I molecules together with HLA-G. Am J Reprod Immunol 37:161-171.

Hazell LJ, Arnold L, Flowers D, Waeg G, Malle E, and Stocker R (1996). Presence of hypochlorite-modified proteins in human atherosclerotic lesions. J Clin Invest 97:1535-1544.

Hazell LJ, Davies MJ, and Stocker R (1999). Secondary radicals derived from chloramines of apolipoprotein B-100 contribute to $\mathrm{HOCl}$-induced lipid peroxidation of low-density lipoproteins. Biochem J 339:489-495.

Hazen SL, Zhang R, Shen Z, Wu W, Podrez EA, MacPherson JC, Schmitt D, Mitra SN, Mukhopadhyay C, Chen Y, Cohen PA, Hoff HF, and Abu-Soud HM (1999). Formation of nitric oxide-derived oxidants by myeloperoxidase in monocytes: Pathways for monocyte-mediated protein nitration and lipid peroxidation In vivo. Circ Res 85:950-958.

Hunt JS, Petroff MG, and Burnett TG (2000). Uterine leukocytes: Key players in pregnancy. Semin Cell Dev Biol 11:127-137.

Koeffler HP, Ranyard J, and Pertcheck M (1985). Myeloperoxidase: Its structure and expression during myeloid differentiation. Blood 65:484-491.

Kossenjans W, Eis A, Sahay R, Brockman D, and Myatt $L$ (2000). Role of peroxynitrite in altered fetal-placental vascular reactivity in diabetes or preeclampsia. Am J Physiol (Heart Circ Physiol) 278:H1311-H1319.

Laham N, Brennecke SP, and Rice GE (1999). Interleukin-8 release from human gestational tissue explants: Effects of gestation, labor, and chorioamnionitis. Biol Reprod 61:823827.

Lang I, Hartmann M, Blaschitz A, Dohr G, Kaufmann P, Frank HG, Hahn T, Skofitsch G, and Desoye G (1994). Differential lectin binding to the fibrinoid of human full-term placenta: Correlation with a fibrin antibody and the PAF-Halmi method. Acta Anat (Basel) 150:170-177.

Lefkowitz SS, Gelderman MP, Lefkowitz DL, Moguilevsky N, and Bollen A (1996). Phagocytosis and intracellular killing of Candida albicans by macrophages exposed to myeloperoxidase. J Infect Dis 173:1202-1207.
Loke YW and King A (1995). Uterine mucosal leucocytes. In: Loke YW and King A, editors. Human implantation - cell biology and immunology. Cambridge, United Kingdom: Cambridge University Press, 102-129.

Loke YW and King A (2000). Decidual natural-killer-cell interaction with trophoblast: Cytolysis or cytokine production? Biochem Soc Trans 28:196-198.

Lyttle CR and DeSombre ER (1977). Uterine peroxidase as a marker for estrogen action. Proc Natl Acad Sci U S A 74:3162-3166.

Malle E, Hazell L, Stocker R, Sattler W, Esterbauer H, and Waeg G (1995). Immunologic detection and measurement of hypochlorite-modified LDL with specific monoclonal antibodies. Arterioscler Thromb Vasc Biol 15:982-989.

Malle E, Waeg G, Schreiber R, Gröne E, Sattler W, and Gröne $\mathrm{HJ}$ (2000). Immunological evidence for the myeloperoxidase/ $\mathrm{H}_{2} \mathrm{O}_{2}$ /halide system in human atherosclerotic lesions. Colocalization of myeloperoxidase and hypochlorite-modified proteins. Eur J Biochem 267:4495-4503.

Malle E, Woenckhaus C, Waeg G, Esterbauer H, Gröne E, and Gröne HJ (1997). Immunological evidence for hypochlorite-modified proteins in human kidney. Am J Pathol 150:603-615.

Many A, Hubel CA, Fisher SJ, Roberts JM, and Zhou Y (2000). Invasive cytotrophoblasts manifest evidence of oxidative stress in preeclampsia. Am J Pathol 156:321-331.

Marcinkiewicz J (1997). Neutrophil chloramines: Missing links between innate and acquired immunity. Immunol Today 18:577-580.

Murphy AA, Palinski W, Rankin S, Morales AJ, and Parthasarathy $S$ (1998). Evidence for oxidatively modified lipid-protein complexes in endometrium and endometriosis. Fertil Steril 69:1092-1094.

Myatt L, Rosenfield RB, Eis AL, Brockman DE, Greer I, and Lyall F (1996). Nitrotyrosine residues in placenta. Evidence of peroxynitrite formation and action. Hypertension 28:488493.

Nakatsuka M, Asagiri K, Kimura Y, Kamada Y, Tada K, and Kudo T (1999). Generation of peroxynitrite and apoptosis in placenta of patients with chorioamnionitis: Possible implications in placental abruption. Hum Reprod 14:1101-1106.

Ogino T, Ma Y, Than TA, Omori M, and Okada S (2000). Monochloramine enhances Fas (APO-1/CD95)-induced apoptosis in Jurkat T cells. J Leukoc Biol 67:46-52.

Osmers RG, Blaser J, Kuhn W, and Tschesche H (1995). Interleukin-8 synthesis and the onset of labor. Obstet Gynecol 86:223-229.

Petty MA, Kintz J, and DiFrancesco GF (1990). The effects of taurine on atherosclerosis development in cholesterol-fed rabbits. Eur J Pharmacol 180:119-127.

Plessinger MA, Woods JR Jr, and Miller RK (2000). Pretreatment of human amnion-chorion with vitamins $C$ and $E$ prevents hypochlorous acid-induced damage. Am J Obstet Gynecol 183:979-985.

Rodesch F, Simon P, Donner C, and Jauniaux E (1992). Oxygen measurements in endometrial and trophoblastic tissues during early pregnancy. Obstet Gynecol 80:283-285.

Sata M and Walsh K (1998). Oxidized LDL activates fasmediated endothelial cell apoptosis. J Clin Invest 102:16821689. 
Savenkova ML, Mueller DM, and Heinecke JW (1994). Tyrosyl radical generated by myeloperoxidase is a physiological catalyst for the initiation of lipid peroxidation in low density lipoprotein. J Biol Chem 269:20394-20400.

Scheuer H, Gwinner W, Hohbach J, Gröne EF, Brandes RP, Malle E, Olbricht CJ, Walli AK, and Gröne HJ (2000). Oxidant stress in hyperlipidemia-induced renal damage. Am J Physiol (Renal Physiol) 278:F63-F74.

Shepherd VL and Hoidal JR (1990). Clearance of neutrophilderived myeloperoxidase by the macrophage mannose receptor. Am J Respir Cell Mol Biol 2:335-340.

Takehara Y, Yoshioka T, and Sasaki J (1990). Changes in the levels of lipoperoxide and antioxidant factors in human placenta during gestation. Acta Med Okayama 44:103-111.

Thomas EL, Grisham MB, and Jefferson MM (1986). Cytotoxicity of chloramines. Methods Enzymol 132:585-593.
Walsh SW, Vaughan JE, Wang Y, and Roberts LJ 2nd (2000). Placental isoprostane is significantly increased in preeclampsia. FASEB J 14:1289-1296.

Watson AL and Burton GJ (1998). A microscopical study of wound repair in the human placenta. Microsc Res Tech 42:351-368.

Weiss SJ, Klein R, Slivka A, and Wei M (1982). Chlorination of taurine by human neutrophils. Evidence for hypochlorous acid generation. J Clin Invest 70:598-607.

Woenckhaus C, Kaufmann A, Bussfeld D, Gemsa D, Sprenger H, and Gröne HJ (1998). Hypochlorite-modified LDL: Chemotactic potential and chemokine induction in human monocytes. Clin Immunol Immunopathol 86:27-33. 